\title{
18 Fünfter Exkurs Mathematik*
}

\subsection{Raumzeigertransformation*}

Dreiphasensysteme (wie in der Drehstromtechnik) können anstatt durch drei verbundene Ausdrücke (z.B. für die Stränge) als ein Raumzeiger in der komplexen Ebene (also mit zwei Komponenten) dargestellt werden. Dabei wird nicht wie in der komplexen Wechselstromrechnung (Abschnitt 11.1.1) eine imaginäre Zeitfunktion hinzugefügt, sondern die drei Phasen werden in der komplexen Ebene räumlich angeordnet. Auch wenn Raumzeiger ähnlich aussehen und gehandhabt werden, dürfen sie nicht mit diesen Zeigern verwechselt werden. Die Definition des Raumzeigers aus den drei Phasen $\mathrm{uLx}_{\mathrm{L}}(\mathrm{t})$ lautet:

$$
\begin{aligned}
& \underline{\mathrm{U}}=2 / 3\left[\mathrm{uL}_{1}(\mathrm{t})+\mathrm{a} \mathrm{uL}_{2}(\mathrm{t})+\mathrm{a}^{2} \mathrm{uL}_{3}(\mathrm{t})\right] \\
& \text { mit } \mathrm{a}=\mathrm{e}^{\mathrm{j} 2 \pi / 3}=-1 / 2+\mathrm{j} \sqrt{3} / 2 \text { und } \mathrm{a}^{2}=\mathrm{e}^{\mathrm{j} 4 \pi / 3}=\mathrm{e}^{-\mathrm{j} 2 \pi / 3}=-1 / 2-\mathrm{j} \sqrt{3} / 2 .
\end{aligned}
$$

Für ein symmetrisches Dreiphasensystem mit den $u_{\mathrm{L} 1}=\hat{\mathrm{U}} \cos (\omega \mathrm{t}), \mathrm{u}_{\mathrm{L} 2}=\hat{\mathrm{U}} \cos (\omega \mathrm{t}-2 \pi / 3)$ und $\mathrm{u}_{\mathrm{L} 3}=\hat{\mathrm{U}} \cos (\omega \mathrm{t}-4 \pi / 3)$ folgt daraus nach Einsetzen in ( 18.1$)$ und Umformen ${ }^{215}$ :

$$
\underline{\mathrm{U}}=\hat{\mathrm{U}} \mathrm{e}^{\mathrm{j} \omega \mathrm{t}} \text {. }
$$

Die Raumzeigerdarstellung enthält nur zwei Komponenten, in unsymmetrischen Systemen evtl. vorhandene Nullsysteme ${ }^{216}$ gehen verloren. Ein Nullsystem muss also separat behandelt und nach Rücktransformation addiert werden. Die Rücktransformation erfolgt mit ${ }^{215}$ :

$$
\mathrm{u}_{\mathrm{L} 1}(\mathrm{t})=\operatorname{Re}\{\underline{\mathrm{U}}\}, \mathrm{u}_{\mathrm{L} 2}(\mathrm{t})=\operatorname{Re}\left\{\mathrm{a}^{2} \underline{\mathrm{U}}\right\} \text { und } \mathrm{u}_{\mathrm{L} 3}(\mathrm{t})=\operatorname{Re}\{\mathrm{a} \underline{\mathrm{U}}\} .
$$

Praxistipp: Oft ist es sinnvoll, durch einen Übergang zu neuen Koordinaten (z.B. in ein mit $\omega t$ rotierendes Koordinatensystem) die Rechnung zu vereinfachen.

Bei einem symmetrischen System entstehen so z.B. zeitkonstante Raumzeiger. Im allgemein mit $\omega_{2} t$ rotierendem System wird dann $\underline{U} z u \underline{U_{R}}=\underline{U} \mathrm{e}^{-\mathrm{j} \omega_{2} \mathrm{t}}=\hat{\mathrm{U}} \mathrm{e}^{\mathrm{j} \omega \mathrm{t}} \mathrm{e}^{-\mathrm{j} \omega_{2} \mathrm{t}}=\hat{\mathrm{U}} \mathrm{e}^{-\mathrm{j}\left(\omega-\omega_{2}\right) \mathrm{t}}$ und im Spezialfall $\omega_{2}=\omega \mathrm{zu} \underline{\mathrm{U}}=\hat{\mathrm{U}}$. Weiterführendes siehe in (Büh77a S. 33).

\subsection{Drehstrom und -felder in der Raumzeigerdarstellung*}

Bei Bauelementen (Kondensatoren, Spulen) mit einer Spannung $\underline{U}_{R}=\underline{U} e^{j \omega_{2} t}$ muss $\underline{U}_{R}$ nach der Produktenregel abgeleitet werden.

$$
\mathrm{d} \underline{\mathrm{U}} \mathrm{R} / \mathrm{dt}=\mathrm{d} \underline{\mathrm{U}} / \mathrm{dt} \cdot \mathrm{e}^{-\mathrm{j} \omega_{2} \mathrm{t}}-\underline{\mathrm{U}} \cdot \mathrm{je} \mathrm{e}^{-\mathrm{j} \omega_{2} \mathrm{t}} \mathrm{d}\left(\omega_{2} \mathrm{t}\right) / \mathrm{dt}=\mathrm{d} \underline{\mathrm{U}} / \mathrm{dt} \mathrm{e}^{-\mathrm{j} \omega_{2} \mathrm{t}}-\mathrm{j} \underline{\mathrm{U}} \mathrm{R} \mathrm{d}\left(\omega_{2} \mathrm{t}\right) / \mathrm{dt}
$$

bzw.

$$
\mathrm{d} \underline{\mathrm{U}} / \mathrm{dt} \mathrm{e}^{-\mathrm{j} \omega_{2} \mathrm{t}}=\mathrm{d} \underline{\mathrm{U}} \mathrm{R} / \mathrm{dt}+\mathrm{j} \underline{\mathrm{U}} \mathrm{R} \mathrm{d}\left(\omega_{2} \mathrm{t}\right) / \mathrm{dt}=\mathrm{d} \underline{\mathrm{U}} \mathrm{R} / \mathrm{dt}+\mathrm{j} \omega_{2} \underline{\mathrm{U}} \mathrm{R}
$$

Für ein rotierendes Bauelement ist demnach $d \underline{U}_{R} / d t+j \omega_{2} \underline{U}_{R}$ anzusetzen.

Praxistipp: Das gilt auch für Ableitungen von rotierenden Feldern (z.B. für $\left.\mathrm{d} \underline{\Phi}_{\mathrm{kop}} / \mathrm{dt}\right)$.

${ }^{215}$ Mit $\cos \alpha=1 / 2\left(\mathrm{e}^{\mathrm{j} \alpha}-\mathrm{e}^{-\mathrm{j} \alpha}\right)$ und der Bedingung $\mathrm{u}_{\mathrm{L} 1}(\mathrm{t})+\mathrm{u}_{\mathrm{L} 2}(\mathrm{t})+\mathrm{u}_{\mathrm{L} 3}(\mathrm{t})=0$.

${ }^{216}$ In allen drei Phasen identischer Anteil (z.B. in der Praxis ein Strom im Nullleiter); siehe auch ( 19.7 ). 